\title{
THE ASYMPTOTIC BEHAVIOR OF ELEMENTARY SYMMETRIC FUNCTIONS ON A PROBABILITY DISTRIBUTION
}

\author{
V.S. KOZYAKIN \\ Institute for Information Transmission Problems \\ 19 Bolshoi Karetny Lane \\ Moscow 101447, Russia \\ A.V. POKROVSKII ${ }^{1}$ \\ National University of Ireland \\ Institute for Nonlinear Science, Department of Physics \\ University College, Cork, Ireland
}

(Received July, 1999; Revised August, 2000)

The problem on asymptotic of the value

$$
\pi(m, n)=m ! \sigma_{m}(p(1, n), p(2, n), \ldots, p(n, n))
$$

is considered, where $\sigma_{m}\left(x_{1}, x_{2}, \ldots, x_{n}\right)$ is the $m$ th elementary symmetric function of $n$ variables. The result is interpreted in the context of nonequiprobable random mappings theory. tions.

Key words: Random Mappings, Chaotic Numerics, Symmetric Func-

AMS subject classifications: $34 \mathrm{~A} 50, \mathrm{~B} 58 \mathrm{~F} 13$.

\section{Statement of the Problem}

Let

with

$$
p(k, n)=\int_{\frac{k-1}{n}}^{\frac{k}{n}} q(t) d t, \quad k=1,2, \ldots, n
$$

$$
\int_{0}^{1} q(t) d t=1
$$

\footnotetext{
${ }^{1}$ Permanent address: Institute of Information Transmission Problems, Russian Academy of Science, 19 Bolshoi Karetny Lane, Moscow 101447, Russia.
} 
where

$$
q(t)=\alpha \beta(t) t^{\gamma-1} \geq 0, \quad \beta(0)=1, \quad \alpha>0, \quad 0<\gamma \leq 1
$$

and the function $\beta(t)$ is continuous on the interval $[0,1]$.

The problem on asymptotic of the value

$$
\pi(m, n)=m ! \sigma_{m}(p(1, n), p(2, n), \ldots, p(n, n))
$$

is considered, where $\sigma_{m}\left(x_{1}, x_{2}, \ldots, x_{n}\right)$ is the $m$ th elementary symmetric function of $n$ variables. This problem arises in particular in the analysis of the distribution of transient time and other combinatorial characteristics in random nonequiprobable graphs [7] whose distributions have proved essential to the analysis of discretizations of dynamic systems with quasi-chaotic behavior. See $[3,6]$ and references therein.

Theorem 1: The following relations are valid for $n \rightarrow \infty$

$$
\begin{gathered}
\pi(m, n) \rightarrow e^{-\frac{1}{2}\left(\int_{0}^{1} q^{2}(t) d t\right) x^{2}} \text { as } \frac{m}{\sqrt{n}} \rightarrow x, \quad \frac{1}{2}<\gamma \leq 1 ; \\
\pi(m, n) \rightarrow e^{-\frac{1}{2} \alpha^{2} x^{2}} \text { as } \frac{m}{\sqrt{n(\log n)^{-1}}} \rightarrow x, \gamma=\frac{1}{2} ; \\
\pi(m, n) \rightarrow \mathcal{L}(\gamma, \alpha x) \text { as } \frac{m}{n^{\gamma} \rightarrow x, \quad 0<\gamma<\frac{1}{2}}
\end{gathered}
$$

where $\mathcal{L}(\gamma, z)$ is the entire analytic function defined by the infinite product

$$
\mathcal{L}(\gamma, z)=\prod_{k=1}^{\infty}\left\{\left[1+\frac{k^{\gamma}-(k-1)^{\gamma}}{\gamma} z e^{-\frac{k^{\gamma}-(k-1)^{\gamma}}{\gamma} z}\right\} .\right.
$$

For $0<\gamma<\frac{1}{2}$ and for the values $|x|<\frac{\gamma}{\alpha}$ the following representation

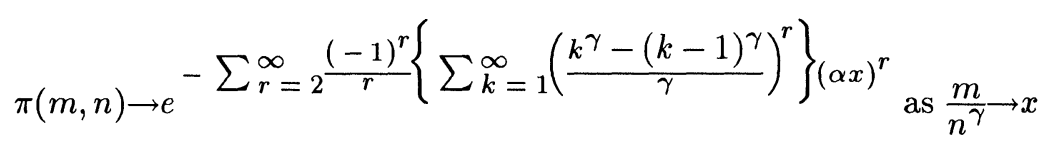

is also valid.

Note a specification of the theorem above in the spirit of random mappings theory [1]. Denote $\Xi(n)=\{1,2, \ldots, n\}$ for a positive integer $n$. Let also the set $\Xi(n)$ be endowed with a probability measure $\mu_{n, q}$ given by equalities $\mu_{n, q}(k)=p(k, n)$, where the function $q$ and the numbers $p(k, n)$ are as in (1). Consider now the random mapping $F_{q, n}$ defined by

$$
\mathbb{P}_{\mu}(F=f)=\prod_{k=1}^{n} p(f(k), n)
$$

Generally speaking, this means that the "appeal" of a point $n$ is proportional to its weight $p(k, n)$. If $q \equiv 1$, then $F_{q, n}$ is a completely random mapping. We emphasize here that the completely random mapping is essentially a purely combinatorial object. The theory of completely random mappings is quite well developed, using 
specifically combinatorial methods. See [1] and the bibliography therein.

For a mapping $f: \Xi(n) \rightarrow \Xi(n)$ and for an element $k \in \Xi(n)$ we denote by $Q(k, f)$ the first recurrence time, that is $Q(k, f)=\min \left\{i: f^{i}(k) f^{j}(k)\right.$, for some $\left.j<i\right\}$. Designate further by $\mathcal{Q}(x, f)$ the scaled distribution function:

$$
\mathcal{Q}(x, f)=\frac{1}{n} \#\left\{k: Q(k, f)<x n^{\gamma}\right\}
$$

where $\# S$ denotes the cardinality of the finite set $S$. The function $\mathcal{Q}$ is a random function if $f$ is considered as realization of the random mapping $F$. Thus, we can consider the mathematical expectation $\mathbb{Q}_{\gamma, n}(x)$. The theorem above implies the following corollary immediately.

Corollary 1: The following relations are valid for $n \rightarrow \infty$

$$
\mathbb{Q}_{\gamma, n}(x) \rightarrow \mathcal{L}(\gamma, \alpha x) \text { as } 0<\gamma<\frac{1}{2} \text {. }
$$

Random mapping with similar asymptotic of weights of elements arises naturally, for instance, in the analysis of discretizations of random mappings where the box counting dimension of the invariant measure differs from its correlation dimension [3].

To conclude this section we note that the equality (5) can be obtained also as a corollary of Theorem 1 [2].

\section{Proof}

The proof of the theorem is based on the following two lemmas:

Lemma 1: Let $f(z)$ and $f_{n}(z), n=1,2, \ldots$, be entire analytic functions of $z \in C$ such that

$$
f_{n}(z) \rightarrow f(z) \text { as } n \rightarrow \infty
$$

uniformly with respect to $z$ from any bounded circle $|z| \leq R$. Let $\mu(n)$ be an integer-valued function and $\nu(n)$ be a real-valued one such that

Then

$$
\nu(n) \rightarrow \infty, \frac{\mu(n)}{\nu(n)} \rightarrow x \text { as } n \rightarrow \infty \text {. }
$$

$$
F(n)=\frac{1}{2 \pi i} \int_{|z|=\rho} \frac{e^{\nu(n) z}}{z^{\mu(n)+1}} f_{n}(z) d z=\frac{(\nu(n))^{\mu(n)}}{\mu(n) !} f(x)(1+o(1)) \text { as } n \rightarrow \infty .
$$

Lemma 2: Let $f(n, z)$ be the polynomial

$$
f(n, z)=\prod_{k=1}^{n}(1+p(k, n) z) .
$$

Then, uniformly with respect to $z$ from any bounded circle $|z| \leq R$ the following relations are valid

$$
\begin{gathered}
f(n, \sqrt{n} z) e^{-\sqrt{n} z} \rightarrow e^{-\frac{1}{2}\left(\int{ }_{0}^{1} q^{2}(t) d t\right) z^{2}} \text { as } n \rightarrow \infty, \frac{1}{2}<\gamma \leq 1 ; \\
f\left(n, \sqrt{n(\log n)^{-1}} z\right) e^{-\sqrt{n(\log n)^{-1}} z} \rightarrow e^{-\frac{1}{2} \alpha^{2} z^{2}} \text { as } n \rightarrow \infty, \gamma=\frac{1}{2} ;
\end{gathered}
$$




$$
f\left(n, n^{\gamma} z\right) e^{-n^{\gamma} z} \rightarrow \mathcal{L}(\gamma, \alpha z) \text { as } n \rightarrow \infty, \quad 0<\gamma<\frac{1}{2}
$$

where $\mathcal{L}(\gamma, z)$ is the entire analytic function defined by $(7)$.

Proof of Theorem 1: Since the proof is identical for the case $0<\gamma<\frac{1}{2}, \gamma=\frac{1}{2}$ and $\frac{1}{2}, \gamma \leq 1$ it will be presented only for the last case.

Remark that the value of $\frac{1}{m !} \pi(m, n)$ by the Viète theorem coincides with the coefficient at the term $z^{m}$ in the Taylor expansion of the function $f(n, z)$ defined by $(9)$. Then, by the theorem on residue of an analytic function

$$
\pi(m, n)=m ! \frac{1}{2 \pi i} \int_{|z|-\rho} \frac{f(n, z)}{z^{m+1}} d z
$$

and, after substitution $z=\sqrt{n} w$,

where

$$
\pi(m, n)=\frac{m !}{(\sqrt{n})^{m}} \frac{1}{2 \pi i} \int_{|w|=\tilde{\rho}} \frac{e^{\sqrt{n} w}}{w^{m+1}} f_{n}(w) d w
$$

$$
f_{n}(w)=f(n, \sqrt{n} w) e^{-\sqrt{n} w} .
$$

From this and from Lemmas 1 and 2 the statement of Theorem 1 for the case $\frac{1}{2}<\gamma \leq 1$ immediately follows.

Although Lemma 1 is in line with statements from the theory of integral's asymptotic proven with the help of the saddle point method (see, e.g., [4, 5]), we failed to find the appropriate reference to the exact formulation. Because of this and for the sake of completeness of presentation, the full proof of Lemma 1 is given below.

Proof of Lemma 1: By the theory on residue of an analytic function

$$
\frac{1}{2 \pi i} \int_{|z|=\rho} \frac{e^{\nu(n) z}}{z^{\mu(n)+1}} d z=\frac{(\nu(n))^{\mu(n)}}{\mu(n) !}
$$

for any value of $\rho>0$. Hence, choosing $\rho=\frac{\mu(n)}{\nu(n)}$, the value of $F(n)$ may be represented as

$$
F(n)=\frac{(\nu(n))^{\mu(n)}}{\mu(n) !} f(x)+\xi(n)
$$

where

$$
\begin{aligned}
\xi(n)= & \frac{1}{2 \pi i} \int \frac{e^{\nu(n) z}}{z^{\mu(n)+1}}\left(f_{n}(z)-f(x)\right) d z . \\
& |z|=\frac{\mu(n)}{\nu(n)}
\end{aligned}
$$

Estimate the value of $|\xi(n)|$. Let us make the substitution $z=\frac{\mu(n)}{\nu(n)} e^{i \varphi}$ in the above integral. Then

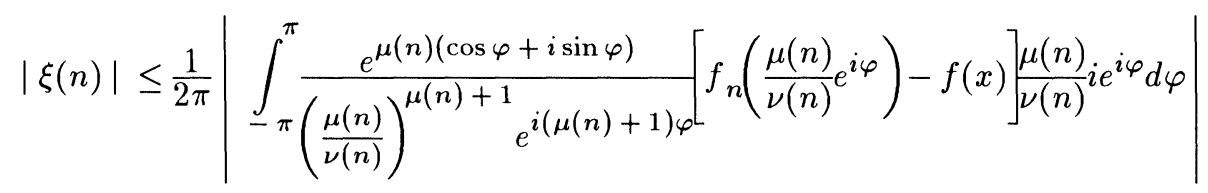




$$
\begin{gathered}
\leq \frac{1}{2 \pi} \int_{-\pi}^{\pi} \frac{e^{\mu(n) \cos \varphi}}{\left(\frac{\mu(n)}{\nu(n)}\right)^{\mu(n)}}\left|f_{n}\left(\frac{\mu(n)}{\nu(n)} e^{i \varphi}\right)-f(x)\right| d \varphi \\
=\frac{1}{2 \pi} \frac{e^{\mu(n)}(\nu(n))^{\mu(n)}}{(\mu(n))^{\mu(n)}} \int_{\pi}^{\pi} e^{\mu(n)(\cos \varphi-1)}\left|f_{n}\left(\frac{\mu(n)}{\nu(n)} e^{i \varphi}\right)-f(x)\right| d \varphi .
\end{gathered}
$$

Now, using the Stirling's formula

$$
\mu(n) !=\frac{1}{\sqrt{2 \pi \mu(n)}}\left(\frac{\mu(n)}{e}\right)^{\mu(n)}\left(1+O\left(\frac{1}{\mu(n)}\right)\right)
$$

we may write that

$$
|\xi(n)| \leq \frac{(\nu(n))^{\mu(n)}}{\mu(n) !} c_{0} \eta(n)
$$

with an appropriate constant $c_{0}$ where

$$
\eta(n)=\sqrt{\frac{\mu(n)}{2 \pi}} \int_{-\pi}^{\pi} e^{\mu(n)(\cos \varphi-1)}\left|f_{n}\left(\frac{\mu(n)}{\nu(n)} e^{i \varphi}\right)-f(x)\right| d \varphi .
$$

In view of (10) and (11), it remains to show that

$$
\eta(n) \rightarrow 0 \text { as } n \rightarrow \infty \text {. }
$$

This proof introduces auxiliary constants

and

$$
\chi_{-\pi \leq \varphi \leq \pi} \frac{2(1-\cos \varphi)}{\varphi^{2}}
$$

$$
c_{1}=\sup _{n \geq 1} \sup _{|z| \leq 2 x}\left|f_{n}(z)-f(x)\right|, \quad \underset{|\varphi|}{|\varphi| \leq \chi^{-1 / 2}(\mu(n))}-1 / 4\left|f_{n}\left(\frac{\mu(n)}{\nu(n)} e^{i \varphi}\right)-f(x)\right| .
$$

Clearly, $\chi>0, c_{1}<\infty$ and $\varepsilon(n) \rightarrow 0$ as $n \rightarrow \infty$ in view of uniform convergence of the sequence $\left\{f_{n}(z)\right\}$ to $f(z)$ on any bounded circle. Hence

$$
\eta(n) \leq \sqrt{\frac{\mu(n)}{2 \pi}} \int_{-\pi}^{\pi} e^{-\frac{\mu(n) \chi}{2} \varphi^{2}}\left|f_{n}\left(\frac{\mu(n)}{\nu(n)} e^{i \varphi}\right)-f(x)\right| d \varphi
$$

and, by substitution $\sqrt{\mu(n) \chi \varphi}=\psi$, we obtain that

$$
\eta(n) \leq \frac{1}{\sqrt{2 \pi \chi}} \int_{\substack{-\sqrt{\mu(n) \chi} \pi}}^{\sqrt{\mu(n) \chi} \pi} e^{-\frac{\psi^{2}}{2}}\left|f_{n}\left(\frac{\mu(n)}{\nu(n)} e^{i \frac{\psi}{\sqrt{\mu(n) \chi}}}\right)-f(x)\right| d \psi=I_{1}(n)+I_{2}(n)+I_{3}(n)
$$

where 


$$
\begin{aligned}
& I_{1}(n)=\frac{1}{\sqrt{2 \pi \chi}} \int^{-(\mu(n))^{1 / 4}} e^{-\frac{\psi^{2}}{2}}\left|f_{n}\left(\frac{\mu(n)}{\nu(n)} e^{i \frac{\psi}{\sqrt{\mu(n) \chi}}}\right)-f(x)\right| d \psi \\
& I_{2}(n)=\frac{1}{\sqrt{2 \pi \chi}} \int^{-(\mu(n))^{1 / 4}} e^{-\frac{\psi^{2}}{2}}\left|f_{n}\left(\frac{\mu(n)}{\nu(n)} e^{i \frac{\psi}{\sqrt{\mu(n) \chi} \pi}}\right)-f(x)\right| d \psi \\
& I_{3}(n)=\frac{1}{\sqrt{2 \pi \chi}} \int_{(\mu(n))^{1 / 4}}^{1 / 4} e^{-\frac{\psi^{2}}{2}}\left|f_{n}\left(\frac{\mu(n)}{\nu(n)} e^{i \frac{\psi}{\sqrt{\mu(n) \chi}}}\right)-f(x)\right| d \psi
\end{aligned}
$$

By definition of the value $\varepsilon(n)$, we have

$$
\left|f_{n}\left(\frac{\mu(n)}{\nu(n)} e^{i \frac{\psi}{\sqrt{\mu(n) \chi}}}\right)-f(x)\right| \leq \varepsilon(n) \text { for }|\psi| \leq(\mu(n))^{1 / 4}
$$

and thus

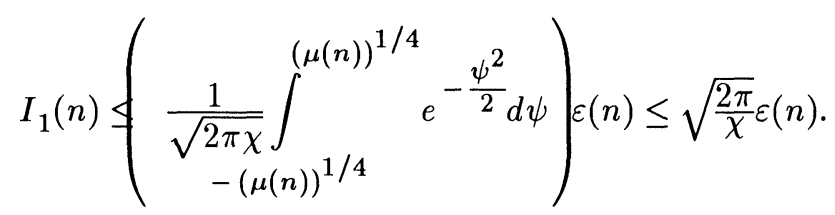

At the same time, by definition of the constant $c_{1}$, we have

$$
I_{2}(n), I_{3}(n) \leq\left(\frac{1}{\sqrt{2 \pi \chi}} \int_{(\mu(n))^{1 / 4}}^{\sqrt{\mu(n) \chi} \pi} e^{-\frac{\psi^{2}}{2}} d \psi\right) c_{1}=\zeta(n) \rightarrow 0 \text { as } n \rightarrow \infty .
$$

From (13), (14) it follows (12) which completes the proof of the lemma.

In the proof of Lemma 2, properties of the numbers $p(k, n)$ play an important role. Therefore, we establish these properties prior to proceeding to the proof of Lemma 2 . From the theorem on mean value of an integral it follows that

$$
p(k, n)=\alpha \beta\left(\tau_{k, n}\right) \frac{k^{\gamma}-(k-1)^{\gamma}}{\gamma n^{\gamma}}, \frac{k-1}{n} \leq \tau_{k, n} \leq \frac{k}{n}, k=1,2, \ldots, n .
$$

Therefore,

$$
p(k, n)=\alpha\left(\beta\left(\frac{k}{n}\right)+\widetilde{\beta}(k, n)\right)^{k^{\gamma}-(k-1)^{\gamma}} \frac{\gamma n^{\gamma}}{\gamma}, k=1,2, \ldots, n
$$

where

$$
\tilde{\beta}(k, n) \leq \max _{\frac{k-1}{n} \leq t \leq \frac{k}{n}}\left|\beta\left(\frac{k}{n}\right)-\beta(t)\right|
$$


and thus, in view of continuity of the function $\beta(t)$,

$$
\widehat{\beta}(n)=\max _{1 \leq k \leq n} \tilde{\beta}(k, n) \rightarrow 0 \text { as } n \rightarrow \infty
$$

From the continuity of the function $\beta(t)$ it follows that the product of the first two multipliers in (15) is uniformly (with respect to $n$ and $k \in[1, n]$ ) bounded. At the same time, for a given $\gamma \in(0,1]$ the numbers $k^{1-\gamma}\left(k^{\gamma}-(k-1)^{\gamma}\right)$ are also uniformly bounded. Then, by (16) such a constant $p_{*}=p(\alpha, \gamma)$ can be chosen that

and simultaneously

$$
\alpha\left(k^{\gamma}-(k-1)^{\gamma}\right) \leq \gamma p_{*} k^{\gamma-1}
$$

$$
0 \leq p(k, n) \leq p_{*} \frac{k^{\gamma-1}}{n^{\gamma}} \leq p_{*} n^{-\gamma} .
$$

Proof of Lemma 2: Case $\frac{1}{2}<\gamma \leq 1$. Set $g(n, z)=f(n, \sqrt{n} z) e^{-\sqrt{n} z}$, then

$$
g(n, z)=e^{-\sqrt{n} z} \prod_{k=1}^{n}(1+p(k, n) \sqrt{n} z)=\prod_{k=1}^{n}\left\{(1+p(k, n) \sqrt{n} z) e^{-p(k, n) \sqrt{n} z}\right\}
$$

(Here, the second equality follows from (1) and (2).) By (19), $0 \leq p(k, n) \sqrt{n} \leq$ $p_{*} n^{1 / 2-\gamma}$ and so $p(k, n) \sqrt{n} \rightarrow 0$ as $n \rightarrow \infty$ uniformly with respect to $k$. Hence, for any $R<\infty$ such a value $n(R)$ can be chosen that $|p(k, n) \sqrt{n} z| \leq \frac{1}{2}$ for $n \geq n(R)$, $|z| \leq R$ uniformly with respect to $k$. Then, for such $n$ and $z$, the representation

$$
\begin{gathered}
1+p(k, n) \sqrt{n} z=e^{\ln (1+p(k, n) \sqrt{n} z)} \\
=e^{p(k, n) \sqrt{n} z-\frac{1}{2}(p(k, n) \sqrt{n} z)^{2}+\theta(k, n, z)(p(k, n) \sqrt{n} z)^{3}}
\end{gathered}
$$

is valid where

$$
|\theta(k, n, z)| \leq \theta_{0}<\infty, n \geq n(R), 1 \leq k \leq n,|z| \leq R
$$

By substituting (21) in (20) we obtain

$$
g(n, z)=e^{-\frac{n}{2}\left(\sum_{k=1}^{n} p^{2}(k, n)\right) z^{2}+\tilde{\theta}(n, z)}
$$

where

$$
\tilde{\theta}(n, z)=n^{\frac{3}{2}}\left(\sum_{k=1}^{n} \theta(, n, z) p^{3}(k, n)\right) z^{3}
$$

From (1) it is seen that $p(k, n)=\frac{1}{n} q\left(t_{k, n}\right)$ with an appropriate $t_{k, n} \in\left[\frac{k-1}{n}, \frac{k}{n}\right]$. Thus, given arbitrary $\varepsilon>0$, we can write

where

$$
\frac{n}{2} \sum_{k=1}^{n} p^{2}(k, n)=S_{1}(\varepsilon, n)+S_{2}(\varepsilon, n)
$$




$$
S_{1}(\varepsilon, n)=\frac{n}{2} \sum_{1 \leq k<\varepsilon n} p^{2}(k, n), S_{2}(\varepsilon, n)=\frac{1}{2} \sum_{\varepsilon n \leq k \leq n} \frac{1}{n} q^{2}\left(t_{k, n}\right) .
$$

From (19) it follows that

$$
S_{1}(\varepsilon, n) \leq \frac{1}{2} p_{*}^{2} n^{1-2 \gamma} \sum_{1 \leq k<\varepsilon n} k^{2(\gamma-1)} \leq \frac{1}{2} c_{0} p_{*}^{2} n^{1-2 \gamma}(\varepsilon n)^{2(\gamma-1)+1}
$$

with an appropriate constant $c_{0}$, and thus

$$
S_{1}(\varepsilon, n) \leq \frac{1}{2} c_{0} p_{*}^{2} \varepsilon^{2 \gamma-1} \rightarrow 0 \text { as } \varepsilon \rightarrow 0 .
$$

On the other hand, in view of the summability of the function $q^{2}(t)$ on the interval $[\varepsilon, 1]$

$$
S_{2}(\varepsilon, n) \rightarrow \frac{1}{2} \int_{\varepsilon}^{1} q^{2}(t) d t \text { as } n \rightarrow \infty
$$

From this and from (25) it follows that

$$
\frac{n}{2} \sum_{k=1}^{n} p^{2}(k, n) \rightarrow \frac{1}{2} \int_{0}^{1} q^{2}(t) d t \text { as } n \rightarrow \infty .
$$

At the same time from (19), (22) and (24) it follows that

$$
\begin{gathered}
|\tilde{\theta}(n, z)| \leq n^{\frac{3}{2}}\left(\sum_{k=1}^{n}|\theta(k, n, z)| p^{3}(k, n)\right) z^{3} \\
\leq \theta_{0} p_{*}^{3} n^{3\left(\frac{1}{2}-\gamma\right)}\left(\sum_{k=1}^{n} k^{3(\gamma-1)}\right) z^{3} \leq \theta_{0} c_{1} p_{*}^{3} n^{3\left(\frac{1}{2}-\gamma\right)} n^{3(\gamma-1)+1} z^{3}
\end{gathered}
$$

with an appropriate constant $c_{1}$. Thus,

$$
|\tilde{\theta}(n, z)| \leq \theta_{0} c_{1} p_{*}^{3} n^{-\frac{1}{2}}
$$

From $(23),(26),(27)$ and from the definition of the function $g(n, z)$ the statement of the lemma for the case $\frac{1}{2}<\gamma \leq 1$ follows:

Case $\gamma=\frac{1}{2}$. Set $g(n, z)=f\left(n, \sqrt{n(\log n)^{-1}} z\right) e^{-\sqrt{n(\log n)^{-1}} z}$, then by (1) and (2)

$$
g(n, z)=\prod_{k=1}^{n}\left\{\left(1+p(k, n) \sqrt{n(\log n)^{-1}} z\right) e^{-p(k, n) \sqrt{n(\log n)^{-1}} z}\right\}
$$

By $(19), 0 \leq p(k, n) \sqrt{n(\log n)^{-1}} \leq p_{*}(\log n)^{-1 / 2}$ and so $p(k, n) \sqrt{n(\log n)^{-1}} \rightarrow 0$ as $n \rightarrow \infty$ uniformly with respect to $k$. Hence, for any $R<\infty$ such a value $n(R)$ can be chosen that $\left|p(k, n) \sqrt{n(\log n)^{-1}} z\right| \leq \frac{1}{2}$ for $n \geq n(R), \quad|z| \leq R$ uniformly with respect to $k$. Then, for such $n$ and $z$ the representation

$$
1+p(k, n) \sqrt{n(\log n)^{-1}} z=e^{\ln \left(1+p(k, n) \sqrt{n(\log n)^{-1}} z\right)}
$$




$$
=e^{p(k, n) \sqrt{n(\log n)^{-1}} z-\frac{1}{2}\left(p(k, n) \sqrt{n(\log n)^{-1}} z\right)^{2}+\theta(k, n, z)\left(p(k, n) \sqrt{n(\log n)^{-1}} z\right)^{3}}
$$

is valid where

$$
|\theta(k, n, z)| \leq \theta_{0}<\infty, n \geq n(R), 1 \leq k \leq n,|z| \leq R
$$

By substituting (29) in (28) we obtain that

where

$$
g(n, z)=e^{-\frac{n}{2 \log n}\left(\sum_{k=1}^{n} p^{2}(k, n)\right) z^{2}+\tilde{\theta}(n, z)}
$$

$$
\tilde{\theta}(n, z)=\left(\frac{n}{\log n}\right)^{\frac{3}{2}}\left(\sum_{k=1}^{n} \theta(k, n, z) p^{3}(k, n)\right) z^{3} .
$$

Estimation of $\tilde{\theta}(n, z)$ is done analogously to the previous case. From (19) and (30) it follows that

$$
\begin{aligned}
&|\tilde{\theta}(n, z)| \leq\left(\frac{n}{\log n}\right)^{\frac{3}{2}}\left(\sum_{k=1}^{n}|\theta(k, n, z)| p^{3}(k, n)\right) z^{3} \\
& \leq \theta_{0} p_{*}^{3}(\log n)^{-\frac{3}{2}}\left(\sum_{k=1}^{n} k^{-\frac{3}{2}}\right) z^{3} \\
& \leq \theta_{0} c_{1} p_{*}^{3} n^{-\frac{1}{2}}(\log n)^{-\frac{3}{2}} z^{3} \rightarrow 0 \text { as } n \rightarrow \infty
\end{aligned}
$$

Before estimating the first summand in the power of the number $e$ in (31), we recall Euler's formula:

from which it immediately follows

$$
\lim _{n \rightarrow \infty}\left(\sum_{m=1}^{n} \frac{1}{m}-\log n\right)=C
$$

$$
\left|\sum_{m=k+1}^{n} \frac{1}{m}-\log \frac{n}{k}\right| \leq C_{*}, \quad 1 \leq k \leq n
$$

with an appropriate constant $C_{*}$.

Now, fix an $\varepsilon>0$ and choose such a real number $\delta>0$ that

$$
\left|1-\beta^{2}(t)\right|=\left|\beta^{2}(0)-\beta^{2}(t)\right| \leq \varepsilon, 0 \leq t \leq \delta .
$$

(This can be done by continuity of the function $\beta(t)$.) Then, we can write

$$
\frac{n}{2 \log n} \sum_{k=1}^{n} p^{2}(k, n)=S_{1}(\delta, n)+S_{2}(\delta, n)
$$

where

$$
S_{1}(\delta, n)=\frac{n}{2 \log n} \sum_{1 \leq k \leq \delta n} p^{2}(k, n), \quad S_{2}(\delta, n)=\frac{n}{2 \log n} \sum_{\delta n<k \leq n} p^{2}(k, n) .
$$

From (19) and (33) it follows that

$$
\begin{aligned}
S_{2}(\delta, n) & \leq \frac{1}{2 \log n} p_{*}^{2} \sum_{\delta n<k \leq n} \frac{1}{k} \leq \frac{1}{2 \log n} p_{*}^{2}\left(C_{*}+\log \frac{n}{\delta n}\right) \\
& \leq \frac{1}{2 \log n} p_{*}^{2}\left(C_{*}+|\log \delta|\right) \rightarrow 0 \text { as } n \rightarrow \infty
\end{aligned}
$$


At the same time by (15)

Thus

$$
S_{1}(\delta, n)=\frac{\alpha^{2}}{2 \log n} \sum_{1 \leq k \leq \delta n} 4 \beta^{2}\left(\tau_{k, n}\right)(\sqrt{k}-\sqrt{k-1})^{2} \text { with } \tau_{k, n} \leq \delta .
$$

where

$$
\begin{gathered}
S_{1}(\delta, n)=\frac{\alpha^{2}}{2}+S_{11}(\delta, n)+S_{12}(\delta, n)+S_{13}(\delta, n) \\
S_{11}(\delta, n)=\frac{\alpha^{2}}{2 \log n}\left(\sum_{1 \leq k \leq \delta n} \frac{1}{k}-\log n\right), \\
S_{12}(\delta, n)=\frac{\alpha^{2}}{2 \log n} \sum_{1 \leq k \leq \delta n} 4\left(\beta^{2}\left(\tau_{k, n}\right)-1\right)(\sqrt{k}-\sqrt{k-1})^{2}, \\
S_{13}(\delta, n)=\frac{\alpha^{2}}{2 \log n} \sum_{1 \leq k \leq \delta n}\left\{4(\sqrt{k}-\sqrt{k-1})^{2}-\frac{1}{k}\right\} .
\end{gathered}
$$

From (33) it follows that

From (34) it follows that

$$
\left|S_{11}(\delta, n)\right| \leq \frac{\alpha^{2}\left(c_{*}+|\log \delta|\right)}{2 \log n} .
$$

$$
\begin{gathered}
\left|S_{12}(\delta, n)\right| \leq \frac{\alpha^{2} \varepsilon}{2 \log n} \sum_{1 \leq k \leq \delta n} 4(\sqrt{k}-\sqrt{k-1})^{2} \leq \frac{\alpha^{2} \varepsilon}{2 \log n} \sum_{1 \leq k \leq \delta n} \frac{4}{k} \\
\leq 2 \alpha^{2} \varepsilon \frac{C_{*}+|\log \delta|+\log n}{\log n} .
\end{gathered}
$$

Finally, since

$$
\frac{1}{k} \leq 4(\sqrt{k}-\sqrt{k-1})^{2}=\left(\frac{2}{\sqrt{k}+\sqrt{k-1}}\right)^{2} \leq \frac{1}{k-1}
$$

then

$$
\begin{gathered}
\left|S_{13}(\delta, n)\right| \leq \frac{\alpha^{2}}{2 \log n} \sum_{1 \leq k \leq \delta n}\left|4(\sqrt{k}-\sqrt{k-1})^{2}-\frac{1}{k}\right| \\
\leq \frac{\alpha^{2}}{2 \log n}\left(3+\sum_{k \geq 2}\left(\frac{1}{k-1}-\frac{1}{k}\right)\right)=\frac{2 \alpha^{2}}{\log n} .
\end{gathered}
$$

From (37), (38), (39) and (40) it follows that

$$
\limsup _{n \rightarrow \infty}\left|S_{1}(\delta, n)-\frac{\alpha^{2}}{2}\right| \leq 2 \alpha^{2} \varepsilon
$$

and thus in view of (35) and (36)

$$
\limsup _{n \rightarrow \infty}\left|\frac{n}{2 \log n} \sum_{k=1}^{n} p^{2}(k, n)-\frac{\alpha^{2}}{2}\right| \leq 2 \alpha^{2} \varepsilon
$$

Since $\varepsilon$ is arbitrary, then

$$
\lim _{n \rightarrow \infty} \frac{n}{2 \log n} \sum_{k=1}^{n} p^{2}(k, n)=\frac{\alpha^{2}}{2}
$$

From this and from (31), (32) the statement of the lemma in the case $\gamma=\frac{1}{2}$ follows.

Case $0<\gamma<\frac{1}{2}$. Set $g(n, z)=f\left(n, n^{\gamma} z\right) e^{-n^{\gamma} z}$, then 


$$
\begin{aligned}
& g(n, z)=e^{-n^{\gamma} z} \prod_{k=1}^{n}\left(1+p(k, n) n^{\gamma} z\right) \\
& =\prod_{k=1}^{n}\left\{\left(1+p(k, n) n^{\gamma} z\right) e^{-p(k, n) n^{\gamma} z}\right\} .
\end{aligned}
$$

(Here the latter equality follows from (1) and (2).) Set also

$$
\begin{gathered}
g(m, n, z)=\prod_{k=1}^{m}\left\{\left(1+p(k, n) n^{\gamma} z\right) e^{-p(k, n) n^{\gamma} z}\right\}, \\
\mathcal{L}(m, \gamma, z)=\prod_{k=1}^{m}\left\{\left[1+\frac{k^{\gamma}-(k-1)^{\gamma}}{\gamma} z\right] e^{-\frac{k^{\gamma}-(k-1)^{\gamma}}{\gamma} z}\right\} .
\end{gathered}
$$

Now, using the evident inequality

$$
\left|(1+z) e^{-z}\right| \leq e^{4|z|^{2}}
$$

and inequalities (18), (19) we can estimate the values $|g(m, n, z)|$ and $|\mathcal{L}(m, \gamma, \alpha z)|$ as follows

$$
\begin{gathered}
|g(m, n, z)|,|\mathcal{L}(m, \gamma, \alpha z)| \leq e^{4 p_{*}^{2}\left(\sum_{k=1}^{m} k^{2(\gamma-1)}\right)|z|^{2}} \\
\leq e^{4 p_{*}^{2}\left(\sum_{k=1}^{\infty} k^{2(\gamma-1)}\right)|z|^{2}}
\end{gathered}
$$

Since the power series $\sum_{k=1}^{\infty} k^{2(\gamma-1)}$ is summable for $0<\gamma<\frac{1}{2}$, then

$$
|g(m, n, z)|,|\ell(m, \gamma, \alpha z)| \leq e^{\omega|z|^{2}}
$$

with an appropriate constant $\omega$ uniformly with respect to all possible combinations of $n$ and $m$. Analogously, for the functions

$$
\begin{gathered}
\widehat{g}(m, n, z)=\prod_{k=m+1}^{n}\left\{\left(1+p(k, n) n^{\gamma} z\right) e^{-p(k, n) n^{\gamma} z}\right\}, \\
\widehat{\ell}(m, \gamma, \alpha z)=\prod_{k=m+1}^{\infty}\left\{\left[1+\frac{k^{\gamma}-(k-1)^{\gamma}}{\gamma} z\right] e^{-\frac{k^{\gamma}-(k-1)^{\gamma}}{\gamma} z}\right\}
\end{gathered}
$$

we can get the following estimates

where

$$
|\widehat{g}(m, n, z)-1|,|\widehat{\mathcal{L}}(m, \gamma, \alpha z)| \leq e^{\omega(m)|z|^{2}}-1
$$

$$
\omega(m)=4 p_{*}^{2}\left(\sum_{k=m+1}^{\infty} k^{2(\gamma-1)}\right) \rightarrow 0 \text { as } m \rightarrow \infty .
$$

Represent the difference $g(n, z)-\mathcal{L}(\gamma, \alpha z)$ in the form

$$
\begin{gathered}
g(n, z)-\mathcal{L}(\gamma, \alpha z)=g(m, n, z) \widehat{g}(m, n, z)-\mathcal{L}(m, \gamma, \alpha z) \widehat{\mathcal{L}}(m, \gamma, \alpha z) \\
=(g(m, n, z)-\mathcal{L}(m, \gamma, \alpha z)) \\
+g(m, n, z)(\widehat{g}(m, n, z)-1)+\mathcal{L}(m, \gamma, \alpha z)(\widehat{\mathcal{L}}(m, \gamma, \alpha z)-1)
\end{gathered}
$$


where the integer $m$ will be chosen later. Then, by (44) and (47)

$$
|g(n, z)-\mathcal{L}(\gamma, \alpha z)| \leq|g(m, n, z)-\mathcal{L}(m, \gamma, \alpha z)|+2 e^{\omega|z|^{2}}\left|e^{\omega(m)|z|^{2}}-1\right| .
$$

Now, fix a number $R>0, \varepsilon>0$ and choose such a big integer $m=m(R, \varepsilon)$ that

Then by (48)

$$
2 e^{\omega R^{2}}\left|e^{\omega(m) R^{2}}-1\right|<\varepsilon
$$

$$
\begin{gathered}
|g(n, z)-\mathcal{L}(\gamma, \alpha z)| \leq|g(m, n, z)-\ell(m, \gamma, \alpha z)|+\varepsilon \\
|z| \leq R, n>m(R, \varepsilon) .
\end{gathered}
$$

But, in view of $(16),(17)$

$$
g(m, n, z) \rightarrow \mathcal{L}(m, \gamma, \alpha z) \text { as } n \rightarrow \infty
$$

for any fixed $m$ uniformly with respect to $z$ from any bounded circle. From here and from (49) we get that

$$
\limsup _{n \rightarrow \infty}|g(n, z)-\mathcal{L}(\gamma, \alpha z)| \leq \varepsilon, \quad|z| \leq R
$$

and thus, since $\varepsilon$ is arbitrary,

$$
g(n, z) \rightarrow \mathcal{L}(\gamma, \alpha z) \text { as } n \rightarrow \infty
$$

uniformly with respect to $|z| \leq R$.

The lemma is completely proved and so is the theorem.

\section{References}

[1] Bollobas, B., Random Graphs, Academic Press, London 1985.

[2] O'Cinneide, C. and Pokrovskii, A., Properties of nonequiprobable random transformations, Ann. Appl. Prob. (to appear).

[3] Diamond, P., Kloeden, P., Pokrovskii, A. and Vladimirov, A., Collapsing effects in numerical simulation of a class of chaotic dynamical systems and random mappings with a single attracting center, Physica D 86 (1995), 559-571.

[4] Fedorjuk, M.V., Asymptotic: Integrals and Series, Nauka, Moscow 1987 (in Russian).

[5] Abi-Khuzam, F.F., The asymptotic behavior of a Lindelof functions and its Taylor coefficients, J. of Math. Anal. and Appl. 93 (1983), 495-526.

[6] Klemm, A. and Pokrovskii, A.V., Random mappings with a single absorbing center and combinatorics of discretizations of the logistic mapping, J. of Appl. Math. and Stoch. Anal. 12:3 (1999), 205-221.

[7] Kolchin, A. and Khokhlov, B., On the number of cycles in random nonequiprobable graph, Diskret. Mat. 2:3 (1990), 137-145 (in Russian). 


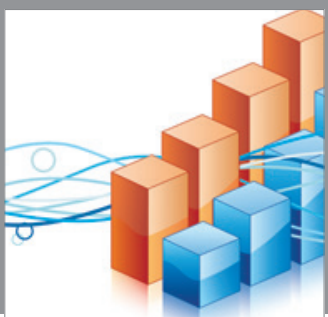

Advances in

Operations Research

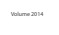

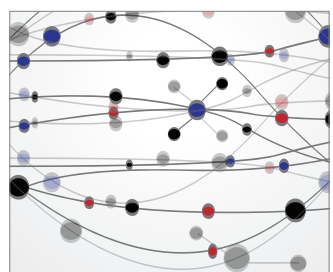

\section{The Scientific} World Journal
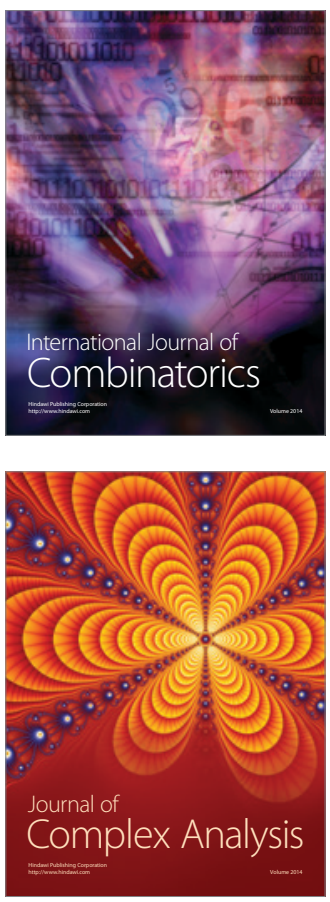

International Journal of

Mathematics and

Mathematical

Sciences
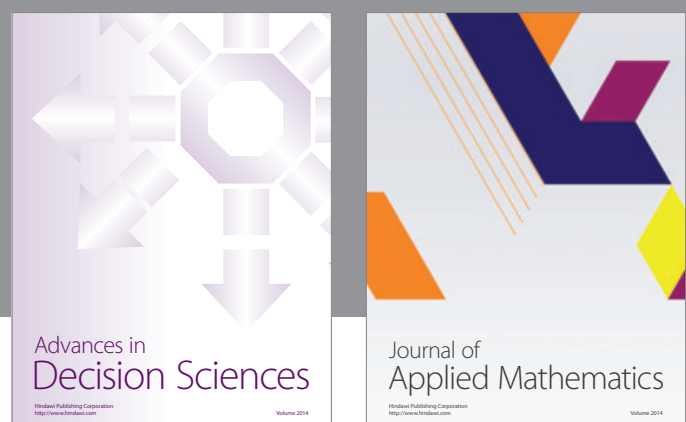

Journal of

Applied Mathematics
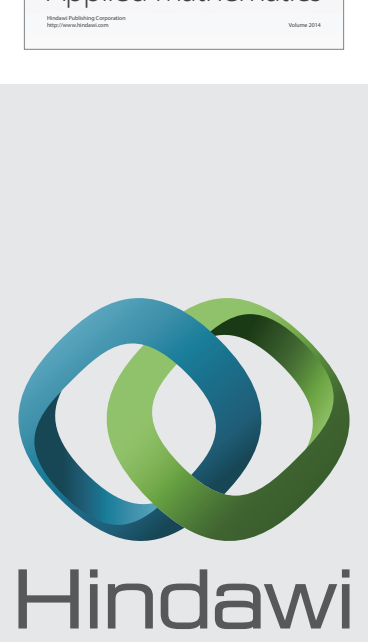

Submit your manuscripts at http://www.hindawi.com
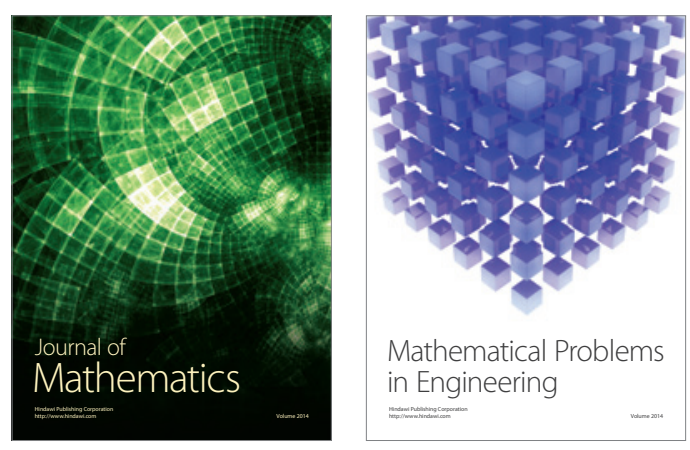

Mathematical Problems in Engineering
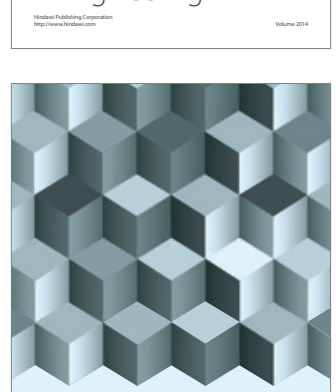

Journal of

Function Spaces
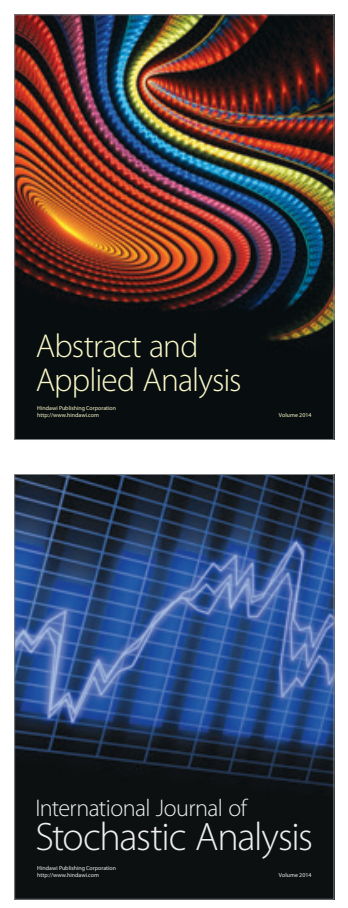

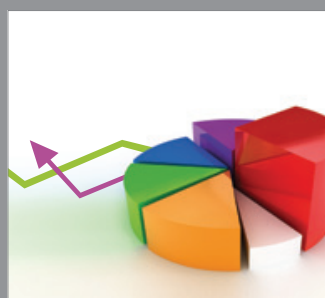

ournal of

Probability and Statistics

Promensencen
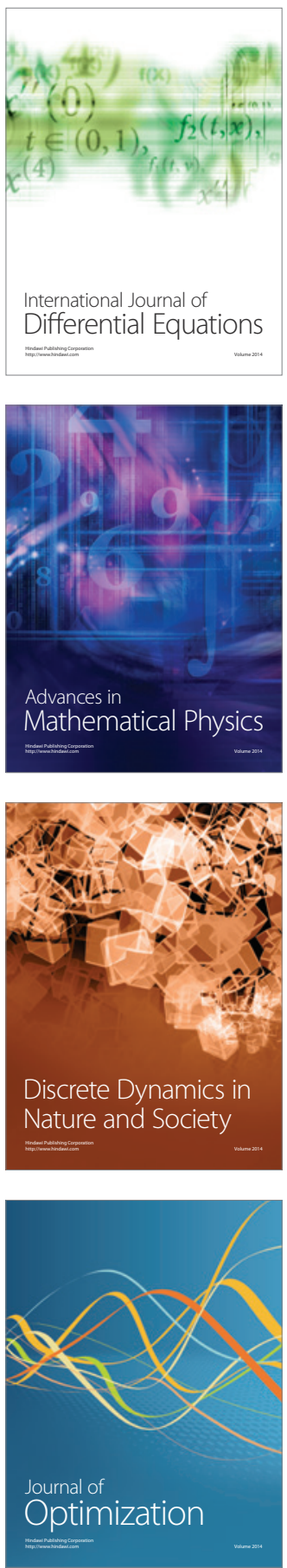